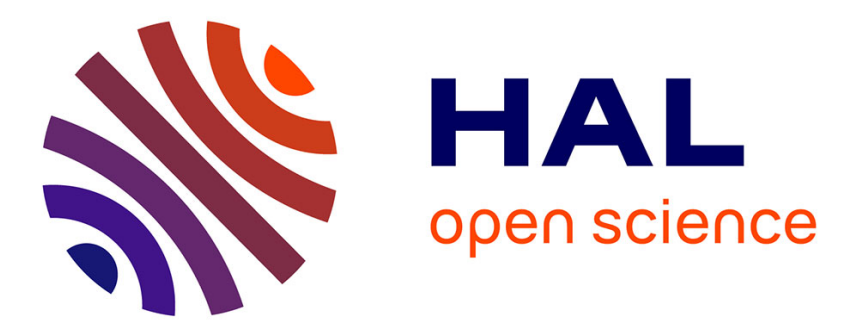

\title{
Active control in an anechoic room: Theory and first simulations
}

D. Habault, E. Friot, Philippe Herzog, C. Pinhede

\section{To cite this version:}

D. Habault, E. Friot, Philippe Herzog, C. Pinhede. Active control in an anechoic room: Theory and first simulations. Acta Acustica united with Acustica, 2017, 103 (3), pp.369-378. 10.3813/AAA.919066 . hal-02337114

\section{HAL Id: hal-02337114 \\ https://hal.science/hal-02337114}

Submitted on 29 Oct 2019

HAL is a multi-disciplinary open access archive for the deposit and dissemination of scientific research documents, whether they are published or not. The documents may come from teaching and research institutions in France or abroad, or from public or private research centers.
L'archive ouverte pluridisciplinaire HAL, est destinée au dépôt et à la diffusion de documents scientifiques de niveau recherche, publiés ou non, émanant des établissements d'enseignement et de recherche français ou étrangers, des laboratoires publics ou privés. 


\title{
Active control in an anechoic room : Theory and first simulations
}

\author{
D.Habault, E.Friot, Ph.Herzog, C.Pinhede \\ Laboratoire de Mécanique et d'Acoustique, \\ Aix Marseille Univ, CNRS, Centrale Marseille, \\ 4 impasse N.Tesla, CS40006, 13453 Marseille Cedex13, France
}

\begin{abstract}
SUMMARY
Noise control and source design require the measurement of sound radiation at low frequencies. Anechoic rooms, which are designed for this purpose, allow echo-free measurements at medium or high frequency but passive wall treatment is less effective at low frequency and in practice no facility provides anechoicity below $50 \mathrm{~Hz}$. This paper discusses the applicability of an active control algorithm which has been previously introduced to minimize the echoes from a scattering object to the cancellation of the low frequency wall echoes in an anechoic room including wall-embedded secondary sources. At first the paper discusses, in the general case then for a free half-space as a model case, the algorithm key which consists in estimating the scattered acoustic pressure from total pressure measurements. Boundary Element Method computations are secondly used to simulate estimation and active control of error signals accounting for the low-frequency scattered pressure in an anechoic room. The simulations show that control with a few dozen microphones and noise sources allows a large reduction of the noise scattered from the walls at low-frequency.
\end{abstract}

\section{Introduction}

Lower audible frequencies have received an increased interest during the last decades, especially because light structures are spreading in many application fields. Annoyance problems are then rising, requiring suitable measurement means and standards. Conversely, existing measurement facilities are barely adapted to the measurement of sources below $70-100 \mathrm{~Hz}$, as conventional lining depth cannot be increased enough for technical and economical reasons. It is thus well known that, at lower frequencies, some unwanted modes of an anechoic room may appear [1]. As an example, measurement of usual audio loudspeakers is a real concern [2]. Although alternatives to free-field measurements have been proposed [3] and are becoming available as commercial solutions [4], most standards still recommend the use of an anechoic room for response measurements [5].

Alternatives to thick lining involving fibrous or porous materials have thus been proposed, among which active control seemed a good candidate as its efficient frequency range complements the one of conventional materials. The use of basic active noise control for the reduction of wall reflections is an old topic, proposed several decades ago for underwater acoustics [6] and audio use [7]. This concept has then been thoroughly validated and improvements have been proposed $[8,9,10,11,12,13]$. Related work is still in progress, the main concerns being to implement a practical sensing method and widen the application range. 
Basic active noise control is however limited to lower frequencies, and becomes less efficient when frequency increases. So-called "hybrid" absorbers have thus been studied, combining absorbing materials and transducers driven so that the resulting device exhibits the performances of a conventional passive lining at higher frequencies and suitable performances at lower frequencies. Such systems have also been thoroughly investigated, with varying combinations of passive material, actuator, and control strategies [14, 15, 16, 17, 18, 19, 20].

Most of the above-mentioned results deal with plane-wave field inside a waveguide, with a normal incidence angle. As pointed out in [21], the absorption coefficient estimated in a planewave tube is a confusing indicator, barely representative of actual absorption performances inside a room. Some works thus addressed this aspect, considering the influence of incidence angles or the behavior of 3D volumes. For example, a speaker array was used to control an oblique incident plane wave [22], dipolar sources were used to damp the first modes of a small room [23], an array of hybrid cells was studied under various incidences [24], or an hybrid cell has been used to dampen a structural-acoustic coupled system [25].

Although one might feel intuitively that lining the walls of a room with "perfect" absorbers should approximate an anechoic situation, there is no direct relation between achieving a locally reacting absorbing condition and anechoicity. This has been simulated by driving hybrid cells from a local or global error criterion, showing that maximum absorption by a local criterion does not achieve a true anechoic condition [26]. This motivated our work aiming at the active control of the pressure reflected by the walls of a $3 \mathrm{D}$ volume, taking into account a non-local boundary condition. This is a problem similar to the control of the field diffracted by an object in free field - but here the "object" surrounds a volume.

The acoustic facets of active control are introduced in text-books such as [27] which specifically includes a chapter dedicated to the control of diffracted sound fields. This chapter discusses earlier references and control strategies that mainly focused at reducing the echoes to sonar excitation. Some recent references also address scattering by an obstacle in a fluid medium $[28,29,30,31,32]$. A main difficulty is then to get a reliable estimate of the scattered field, a non-measurable quantity. For example, the diffracted pressure may be deduced from two series of measurements, with and without the diffracting object.

For the application to an anechoic room, the diffracted pressure is the pressure reflected by the walls of the room so that such a method cannot be applied. An integral relation may be used to estimate a quantity to be minimized (the scattered field) from the total pressure observed with usual pressure microphones [33, 34]. This mathematical operator is considered here as a matrix of filters to be applied to the error microphones. For practical purposes, this matrix is estimated during a calibration step, using a set of reference sources and an array of identification microphones. As a first test of the method, an experiment was carried out in a scaled mock-up of an actual room [35, 36].

The aim of the present paper is twofold. First, some theoretical developments are provided in order to characterize the relation between the total and the diffracted pressure. Secondly, the efficiency of the method is shown on a numerical example of control. The paper is organized as follows. Section 2 presents the details of the method. Section 3 is dedicated to the study of the 


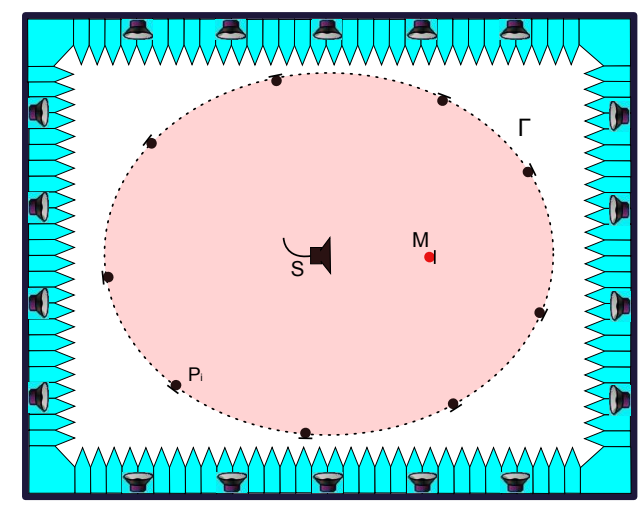

FigURE 1 - Active control of the sound pressure in an anechoic room

operator used to relate the total pressure measured on the array of identification microphones and the diffracted pressure measured at any minimization point. Section 4 presents a example of control. Section 5 presents the conclusion of the study at this stage.

\section{Description of the method}

The geometry considered in this paper is shown in Figure 1. In what follows, it is assumed that the walls of the room provide some acoustic absorption. The method is based on the assumption that it is possible to write a relation between $p_{t o t}$ the total pressure measured on a surface $\Gamma$ and $p_{\text {dif }}$ the diffracted pressure received at an observation point $M$ located in the volume $\Omega$ inside $\Gamma$. This relation is expressed with the help of an operator or a "filter" $\mathcal{H}$ as :

$$
p_{\text {dif }}(S, M)=\int_{\Gamma} p_{\text {tot }}(S, P) \mathcal{H}(M, P) d \sigma(P)
$$

The observation point $M$ may represent any of the minimization microphones as defined in the previous section. The surface $\Gamma$ represents the surface described by the set of the identification microphones. The relation will be applied to $S$ the primary source, that is the source to be characterized in the room.

The existence of such a relation is not so conventional. Indeed, it is well known that the diffracted pressure may be written as an integral of the total pressure and the normal derivative of the total pressure on any surface inside the room by using integral representations. The point of interest here is to express the diffracted pressure as a function of the total pressure only. If the relation exists and if the operator $\mathcal{H}$ is known, the total pressure measured on $\Gamma$ can be used to drive the secondary sources of the active control system and reduce the diffracted pressure at point $M$. In practice, the integral relation is discretized and the surface $\Gamma$ is approximated by the set of identification microphones. The method includes two steps. The first one is a precalibration step in which an approximation of the filter $\mathcal{H}$ is evaluated. The second one is a control step in which an active control algorithm is applied to drive the secondary sources.

The precalibration step is based on the essential fact that $\mathcal{H}$ does not depend on the source $S$. It can therefore be evaluated with a set of sources denoted here identification sources which are chosen such that their sound radiation patterns are known. It is determined by minimizing the 
difference between the diffracted pressure at point $M$ and its expression (1). Once a numerical approximation of $\mathcal{H}$ is obtained, the approximation of the integral relation can be used to compute the diffracted pressure for any other source. Then the active control step is carried out.

Previous experimental results showed that such a method provides efficient control results [34]. However it is still necessary to prove the validity of formula (1) and to check the existence and the properties of the operator $\mathcal{H}$. Indeed the quality of the control relies on the properties of this operator. This is the aim of the analysis presented in the next paragraph.

\section{The integral relation between the total pressure and the dif- fracted pressure}

By using conventional Green's representations it is possible to relate the sound pressure inside a volume to the values of this pressure and of its first normal derivative on the boundary of the volume [37]. Writing a relation such as (1) with no normal derivative term is a bit more challenging.

A simple idea is to choose the specific Green's function defined in the volume inside $\Gamma$ which satisfies a homogeneous Dirichlet boundary condition on $\Gamma$. This leads to a relation of the expected form (1). However this Green's function is not uniquely defined for frequencies equal to the Dirichlet resonance frequencies of the volume. Since the aim of the study is to evaluate the mathematical properties of the operator $\mathcal{H}$ it is more efficient to introduce only welldefined (uniquely defined) Green's functions to construct the operator. Therefore the propagation problem is transformed into a transmission problem [38, 39] between two domains : The domain $\Omega_{1}$ corresponding to the inside of the surface $\Gamma$ of the identification microphones and the domain $\Omega_{2}$ located between $\Gamma$ and the walls of the room. The sound pressures defined in each domain are related by continuity conditions on the interface $\Gamma$. The method consists in using two Green's functions defined for any real frequency and applying integral representations to the two sound pressures. Developments are given for the most general case and then for the half-space case for which some analytical expressions based on 2D-Fourier transforms are obtained.

\subsection{The general case}

The transmission problem is considered for a volume with absorbing conditions on the walls.

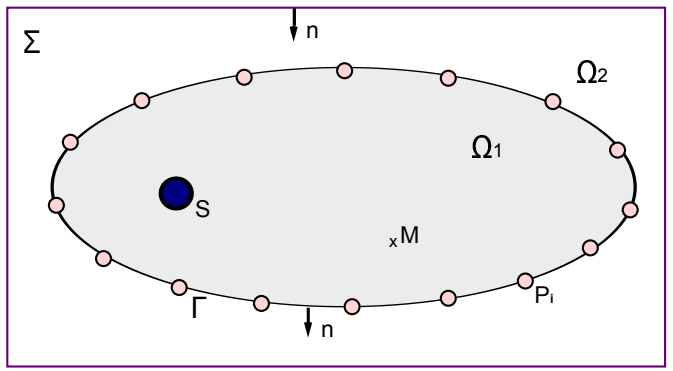

Figure 2 - Geometry of the problem 
The room is divided into two domains, an interior domain $\Omega_{1}$ and an exterior domain $\Omega_{2}$ as shown in Figure 2. The surface $\Gamma$ is the boundary between the two domains and $\Sigma$ represents the outside boundary of $\Omega_{2}$ that is the walls of the room. Two unit normal vectors $\vec{n}$ are defined, both directed towards the interior of $\Omega_{2}$. One is normal to the boundary $\Gamma$ and the other one is normal to the boundary $\Sigma$.

Both domains are characterized by the same sound speed $c$ and density $\rho$. An acoustic source is placed in $\Omega_{1}$ and emits a sinusoidal signal denoted by $e^{-\imath \omega t}$. The sound pressure is denoted $p_{1}$ in $\Omega_{1}$ and $p_{2}$ in $\Omega_{2}$. The problem of sound transmission between the two domains is described by the following system :

$$
\begin{cases}\left(\Delta+k^{2}\right) p_{1}(M)=F & \text { in } \Omega_{1} \\ \left(\Delta+k^{2}\right) p_{2}(M)=0 & \text { in } \Omega_{2} \\ p_{1}=p_{2} & \text { on } \Gamma \\ \partial_{n} p_{1}=\partial_{n} p_{2} & \text { on } \Gamma \\ \mathcal{A}\left(p_{2}, \partial_{n} p_{2}\right)=0 & \text { on } \Sigma\end{cases}
$$

where $k=\omega / c$ is the wavenumber and $\partial_{n} p_{i}$ denotes the normal derivative of $p_{i}$ on the boundary $\Gamma$. At this stage, the operator $\mathcal{A}$ does not need to be specified but it must include an absorbing condition (in a simple case, $\mathcal{A}$ could correspond to an impedance condition for example). Because of this absorbing condition, the solution $\left(p_{1}, p_{2}\right)$ to this system exists and is unique [39, 37, 40].

Let $G_{1}$ be the conventional Green's function defined in the infinite space :

$$
G_{1}\left(M, M^{\prime}\right)=-\frac{e^{\imath k R\left(M, M^{\prime}\right)}}{4 \pi R\left(M, M^{\prime}\right)}
$$

where $R\left(M, M^{\prime}\right)$ is the distance between two points $M$ and $M^{\prime}$.

The Green's representation of $p_{1}$ in $\Omega_{1}$ writes :

$$
\begin{aligned}
& p_{1}(M)=p_{i n c}(M)- \\
& \int_{\Gamma}\left[\partial_{n^{\prime}} p_{1}(P) G_{1}(M, P)-p_{1}(P) \partial_{n^{\prime}} G_{1}(M, P)\right] d P
\end{aligned}
$$

where $p_{\text {inc }}$ is the incident sound pressure. The term $\partial_{n^{\prime}}$ is used for the normal derivative at the integration point $P$ and $d P$ is shortly written for the element of integration. The integral on $\Gamma$ is equal to the diffracted pressure $p_{d i f}(M)$. It can be formally written as :

$$
p_{d i f}(M)=\mathcal{V}_{1}\left[\partial_{n^{\prime}} p_{1}\right]+\mathcal{U}_{1}\left[p_{1}\right]
$$

with the definitions, for any function $\mu$ on $\Gamma$ :

$$
\begin{gathered}
\mathcal{V}_{1}[\mu](M) \equiv-\int_{\Gamma} \mu(P) G_{1}(M, P) d P \\
\mathcal{U}_{1}[\mu](M) \equiv \int_{\Gamma} \mu(P) \partial_{n^{\prime}} G_{1}(M, P) d P
\end{gathered}
$$

In this representation, $\mathcal{U}_{1}$ is a double layer potential operator which operates on the value of the total pressure on $\Gamma$, similarly to the operator $\mathcal{H}$.

The operator $\mathcal{V}_{1}$ corresponds to a simple layer potential and operates on the derivative of the total pressure on $\Gamma$. In order to obtain a formula similar to (1) this first term must be 
transformed into a term which relates the total pressure on $\Gamma$ to the diffracted pressure in $\Omega_{1}$. This is done by writing another relation to link $p_{1}$ and its normal derivative on $\Gamma$.

Let the Green's function $G_{2}$ be defined in $\Omega_{2}$ as the solution to the system of equations :

$$
\begin{cases}\left(\Delta+k^{2}\right) G_{2}\left(M, M^{\prime}\right)=\delta\left(M, M^{\prime}\right) & \text { in } \Omega_{2} \\ G_{2}=0 & \text { on } \Gamma \\ \mathcal{A}\left(G_{2}, \partial_{n} G_{2}\right)=0 & \text { on } \Sigma\end{cases}
$$

Because the condition on $\Sigma$ is an absorbing condition, $G_{2}$ exists and is unique (see for example [40]). Let us recall that explicit analytic expressions of $G_{2}$ are known for a few number of simple conditions and geometries [41].

The Green's formula applied to $G_{2}$ and the pressure $p_{2}$ in $\Omega_{2}$ leads to :

$$
p_{2}(M)=-\int_{\Gamma} p_{2}\left(P^{\prime}\right) \partial_{n^{\prime}} G_{2}\left(M, P^{\prime}\right) d P^{\prime}
$$

because of the boundary condition of $G_{2}$ on $\Gamma$. The next step is to apply a normal derivative to this expression and take the limit when $M$ tends to a point $P$ on $\Gamma$. This requires special attention since it leads to the derivative of a double layer potential which is a highly singular integral but can still be defined properly (see [37, 40]) :

$$
\partial_{n} p_{2}(P)=-\partial_{n(P)} \int_{\Gamma} p_{2}\left(P^{\prime}\right) \partial_{n^{\prime}} G_{2}\left(P, P^{\prime}\right) d P^{\prime}
$$

Introducing the continuity conditions between $p_{1}$ and $p_{2}$ in this equation leads to:

$$
\begin{aligned}
\partial_{n} p_{1}(P) & =-\partial_{n(P)} \int_{\Gamma} p_{1}\left(P^{\prime}\right) \partial_{n^{\prime}} G_{2}\left(P, P^{\prime}\right) d P^{\prime} \\
& \equiv\left[T\left[p_{1}\right]\right](P)
\end{aligned}
$$

where $P$ is a point on $\Gamma$. The second equality defines the operator $T$ which relates the total pressure $p_{1}$ to its derivative $\partial_{n} p_{1}$ both defined on $\Gamma$. The next step is to replace $\partial_{n^{\prime}} p_{1}(P)$ in the first term of the right-hand side in Equation $(3)$ by $T\left[p_{1}\right]$. This leads to :

$$
\begin{aligned}
& \mathcal{V}_{1}\left[\partial_{n} p_{1}\right](M)=\mathcal{V}_{1}\left[T\left[p_{1}\right]\right](M)= \\
& \int_{\Gamma} \partial_{n(P)}\left[\int_{\Gamma} p_{1}\left(P^{\prime}\right) \partial_{n^{\prime}} G_{2}\left(P^{\prime}, P\right) d P^{\prime}\right] G_{1}(M, P) d P
\end{aligned}
$$

Following [37] it is possible to show that the two integrals in this expression can be interchanged. This gives :

$$
\begin{aligned}
& \mathcal{V}_{1}\left[T\left[p_{1}\right]\right]= \\
& \int_{\Gamma} p_{1}\left(P^{\prime}\right) \partial_{n^{\prime}}\left[\int_{\Gamma} G_{1}(M, P) \partial_{n^{\prime}} G_{2}\left(P^{\prime}, P\right) d P\right] d P^{\prime}
\end{aligned}
$$

Using this last equation and Equation (3) shows that the operator can be formally expressed :

$$
\mathcal{H}=-\partial_{n^{\prime}} G_{1}+\partial_{n^{\prime}}\left[\int_{\Gamma} G_{1} \partial_{n^{\prime}} G_{2}\right]
$$


This is a way to obtain an expression of the operator $\mathcal{H}$. Let us point out that the method used here is based on two Green's functions which are defined and unique for any real frequency. In the general case it is not so easy to determine the mathematical properties of the operator. Their study will provide the necessary information on the minimization problem but stands beyond the purpose of the present article. It could be based on the theory of pseudo-integral operators [42]. As shown in the next paragraph, in the simple case of a half-space geometry and an impedance condition, an explicit expression of the operator $\mathcal{H}$ can be obtained. This is of interest since as far as the mathematical non-smoothness of the operator is concerned the properties obtained for the half-space case and for the general case are of the same order.

\subsection{The half-space case}

In the half-space case the total and diffracted sound pressures can be calculated in the Fourier domain in order to get a closer idea of the characteristics of the operator $\mathcal{H}$. Here the surfaces $\Sigma$ and $\Gamma$ corresponding to the walls of the room and to the array of the identification microphones are chosen as the infinite planes $z=0$ and $z=a$ respectively. The problem is solved for a point harmonic source located at $S=(0,0, s>a)$. The minimization points are in the domain $(z>a)$. The sound pressure $p$ in the domain $z>0$ is the solution to the following system :

$$
\begin{cases}\left(\Delta+k^{2}\right) p(M)=\delta(S, M) & \text { in } z>0 \\ \mathcal{A}\left(p, \partial_{n} p\right)=0 & \text { on } \Sigma(z=0) \\ \text { Sommerfeld conditions at infinity } & \end{cases}
$$

Since the propagation medium is homogeneous and isotropic, the pressure only depends on the radial coordinate $\rho=\sqrt{x^{2}+y^{2}}$ and may be written $p(\rho, z)$. The $2 \mathrm{D}$ transverse Fourier transform and its inverse are defined by :

$$
\hat{p}(\xi, z)=2 \pi \int_{0}^{\infty} p(\rho, z) J_{0}(\rho \xi) \rho d \rho
$$

and

$$
p(\rho, z)=\frac{-\imath}{4} \int_{-\infty}^{\infty} \hat{p}(\xi, z) H_{0}^{(1)}(\rho \xi) \xi d \xi
$$

where $J_{0}$ is the Bessel function of zero order and $H_{0}^{(1)}$ the Hankel function of zero order and of the first kind. In the second integral the lower limit $(-\infty)$ must be understood as $\left(\infty e^{2 \pi}\right)$ for a correct definition of the Hankel function [43].

The solution $\hat{p}(\xi, z)$ may be written for $z>0$ :

$$
\hat{p}(\xi, z)=\hat{p}_{t o t}(\xi, z)=\frac{e^{\imath K|z-s|}}{2 \imath K}+\hat{A}(\xi) \frac{e^{\imath K(z+s)}}{2 \imath K}
$$

where $K=\sqrt{k^{2}-\xi^{2}}$ chosen such that $\operatorname{Im}(K)>0$ and $\hat{A}(\xi)$ is the plane wave reflection coefficient. The second term on the right-hand side is the diffracted pressure $\hat{p}_{\text {dif }}(\xi, z)$.

The operator $\hat{\mathcal{H}}$ is equal to the ratio between $\hat{p}_{\text {dif }}(\xi, z)$ and $\hat{p}_{\text {tot }}(\xi, z=a)$ :

$$
\hat{\mathcal{H}}(\xi, z) \equiv \frac{\hat{p}_{d i f}(\xi, z)}{\hat{p}_{\text {tot }}(\xi, a)}=\frac{\hat{A}(\xi) e^{\imath K z}}{e^{-\imath K a}+\hat{A}(\xi) e^{\imath K a}}
$$


Let us remark that $\hat{\mathcal{H}}$ depend on $\xi$ and $a$ and does not depend on the source position $s$ as expected.

For the particular case of an impedance condition on $(z=0) \hat{A}(\xi)$ is explicitly obtained. The impedance condition is written $\partial_{n} p+\imath k p / \zeta=0$ where $\zeta$ represents the reduced specific normal impedance and $\vec{n}$ is the unit vector normal to the plane $(z=0)$ and pointing towards the domain $(z>0)$. Therefore :

$$
\hat{A}(\xi)=\frac{K \zeta-k}{K \zeta+k}
$$

and

$$
\hat{\mathcal{H}}(\xi, z)=e^{\imath K z} \frac{K \zeta-k}{2 K \zeta \cos K a-2 \imath k \sin K a}
$$

The inverse Fourier transform of $\hat{\mathcal{H}}$ can be formally expressed as a sum of layer potentials located in the $(z=a)$ plane, following steps and results presented in [44]. The details of the calculation are given in Appendix. The result may be written :

$$
\begin{aligned}
& \mathcal{H}(Q)=\frac{-1}{2} \frac{\partial}{\partial z}\left(\frac{e^{\imath k r(O, P)}}{4 \pi r(O, P)}\right)-\frac{\imath}{2} \frac{\partial}{\partial z} \int_{z^{\prime}=a} \\
& \sum_{n} c_{n} H_{0}\left(\lambda_{n} \rho\left(P^{\prime}\right)\right) \frac{\partial}{\partial z^{\prime}}\left(\frac{e^{\imath k r\left(Q, P^{\prime}\right)}}{4 \pi r\left(Q, P^{\prime}\right)}\right) d P^{\prime}
\end{aligned}
$$

where $c_{n}=\lambda_{n} h_{b}\left(\lambda_{n}^{2}\right) / D^{\prime}\left(\lambda_{n}^{2}\right)$ with the notations defined in Appendix.

Finally the diffracted pressure is expressed as :

$$
p_{\text {dif }}(Q)=\int_{z=a} p_{\text {tot }}(P) \mathcal{H}(Q-P) d P
$$

Replacing $\mathcal{H}$ by formula (7) leads to an expression similar to the one obtained in the general case with integrals on $\Gamma$. In particular the first term of (8) is :

$$
\frac{-1}{2} \int_{z^{\prime}=a} p_{t o t}(P) \frac{\partial}{\partial z}\left(\frac{e^{\imath k r(Q, P)}}{4 \pi r(Q, P)}\right) d P
$$

\section{An example of control}

As said in the introduction, the method proposed here was first tested experimentally on a scaled model (see $[35,36]$ ). The aim of this section is to present a detailed numerical example which illustrates the way the method applies and to examine its efficiency. The efficiency criterion is taken from the standard rules for anechoic rooms (ISO 3745 and ANSI S12.35 norms). The comparison is made between the sound pressure levels obtained with control along one axis in the room and the corresponding exact incident pressure levels. The anechoicity criterion is said to be fulfilled if the deviations stay within $\pm 1.5 \mathrm{~dB}$.

In this example the "measured" data (incident and diffracted pressure at all microphones) are computed using a BEM code called FELIN [30]. The code is based on the Green's formula and the solutions are obtained by using piecewise-constant approximations on triangular elementary domains. In what follows these data are called "exact".

The next paragraphs present the geometry of the problem, the precalibration step and the control step. The source $\tilde{S}$ represents the source to be characterized (such as the loudspeaker in 


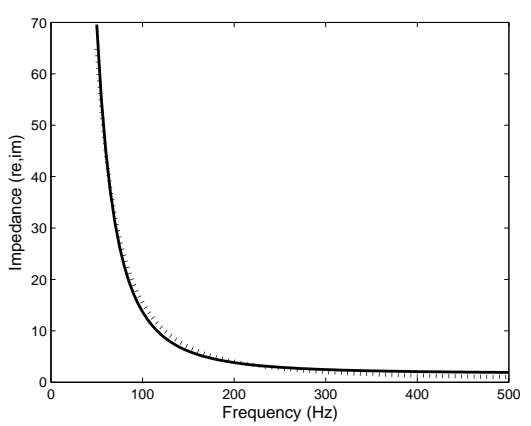

FiguRE 3 - Reduced specific normal impedance versus frequency - Real part (continuous line) and imaginary part $(+)$

example [3]). For the precalibration step, comparisons are presented between the exact diffracted pressure levels computed at a set of minimization points $M_{\ell}$ and those obtained from the identification of the operator $\mathcal{H}$ by using Equation (1). To illustrate the control, comparisons are presented between the exact incident pressure levels emitted by a source $\tilde{S}$ at points $M_{\ell}$ and those obtained after control.

Let us point out that for this example, both steps are carried out in the frequency domain. In practical situations, the precalibration will be carried out in the frequency domain and the active control will be implemented in real-time using the Fx-LMS algorithm [45].

\subsection{The geometry}

The dimensions of the room are those of the scaled model that is a room of dimensions $(2 \mathrm{~m}$ $\times 1.2 \mathrm{~m} \times 1.1 \mathrm{~m})$. The computations described here are carried out for the $50-450 \mathrm{~Hz}$ frequency band. This means that the maximum dimension of the room goes from $0.3 \lambda$ to $2.7 \lambda$.

The precalibration step is achieved with a set of $N_{m}=32$ identification microphones $P_{i}$ and a set of $N_{s}=32$ positions of identification sources $S_{j}$. For the control, 32 loudspeakers $V_{k}$ are mounted in the walls so that they are flush with the walls.

The six walls of the room are described by the same impedance values. Figure 3 presents the curve of the reduced specific normal impedance (real and imaginary parts) as a function of frequency. The choice of these impedance values was not meant to reproduce the behaviour of the walls in an anechoic room but rather to provide a reflection coefficient with very high values at low frequency going down to low values around $450 \mathrm{~Hz}$. Here the amplitude reflection coefficient goes from 0.98 at $50 \mathrm{~Hz}$ to 0.47 at $450 \mathrm{~Hz}$. This is a way to explore several kinds of regimes, to get modes at low frequency and increasing absorption at medium frequency. Indeed, at very low frequency, some modes may be observed in anechoic rooms. A specific study of the effect of the impedance parameter on the identification results shows, not surprisingly, that the best identification of the diffracted pressure is obtained for resonance frequencies that is where the amplitude of the diffracted pressure is large compared with the incident pressure. On the contrary if the diffracted pressure level is low, the identification of the pressure is not that good but in that case an accurate control is not necessary. So this is a positive aspect for the active control. 


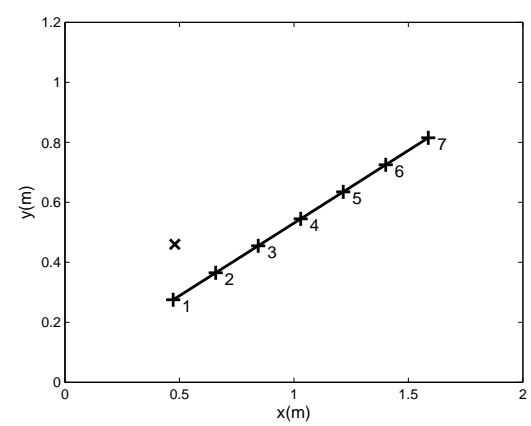

Figure 4 - Source $\tilde{S}(\times)$ and minimization points $(+)$ in the $(z=0.59)$ plane

The source $\tilde{S}$ to be characterized is here a monopole located at $(0.48,0.46,0.47)$ and 7 minimization points $M_{\ell}$ are located on a straight line in the plane $z=0.59$ at a distance from the source going from 0.22 (point 1) to 1.17 (point 7). They are presented in Figure 4. All distances and lengths are given in meters.

\subsection{The identification or precalibration step}

This step consists in estimating the diffracted sound pressure $p_{d i f}\left(\tilde{S}, M_{\ell}\right)$ from the identification of the operator $\mathcal{H}$. Since the operator $\mathcal{H}$ does not depend on the source an approximation is obtained by minimizing the linear system :

$$
F\left(S_{j}, M_{\ell}\right)=\left\|p_{d i f}\left(S_{j}, M_{\ell}\right)-\sum_{i=1}^{N_{m}} p_{t o t}\left(S_{j}, P_{i}\right) \mathcal{H}\left(M_{\ell}, P_{i}\right)\right\|
$$

The identification sources $S_{j}$ are used one by one in turn. Their radiation pattern in free-field is supposed to be known. Here they are chosen as monopoles.

Let us define the two vectors $H$ and $E$ and the matrix $\mathcal{P}$ by :

$$
\begin{array}{ll}
H_{i}=\mathcal{H}\left(M_{\ell}, P_{i}\right) & i=1, N_{m} \\
E_{j}=p_{\text {dif }}\left(S_{j}, M_{\ell}\right) & j=1, N_{s} \\
\mathcal{P}_{i j}=p_{\text {tot }}\left(S_{j}, P_{i}\right) & i=1, N_{m} \text { and } j=1, N_{s}
\end{array}
$$

The element $\mathcal{P}_{i j}$ is the total pressure emitted by the source $S_{j}$ alone and measured at microphone $P_{i} . E_{j}$ is the diffracted pressure due to the source $S_{j}$ alone and received at point $M$. This quantity cannot be measured in a straightforward way; it is computed as the difference $\left(p_{t o t}\left(S_{j}, M\right)-\right.$ $\left.p_{\text {inc }}\left(S_{j}, M\right)\right)$ where the first term is measured and the second term is known as soon as $S_{j}$ is known.

This optimization problem is solved by a Singular Value Decomposition method (SVD) [46]. Once $H$ is known, the estimate denoted $p_{\text {dest }}$ of the diffracted pressure can be computed for any source $\tilde{S}$ inside the boundary $\Gamma$ by formula (1) applied to the source $\tilde{S}$.

The positions of the identification microphones and sources are shown in Figure 5. The microphones $P_{i}$ are regularly spaced on a rectangular array which is $0.15 \mathrm{~m}$ away from the walls of the room. The shortest distance between two microphones is $0.56 \mathrm{~m}$ (except close to the 


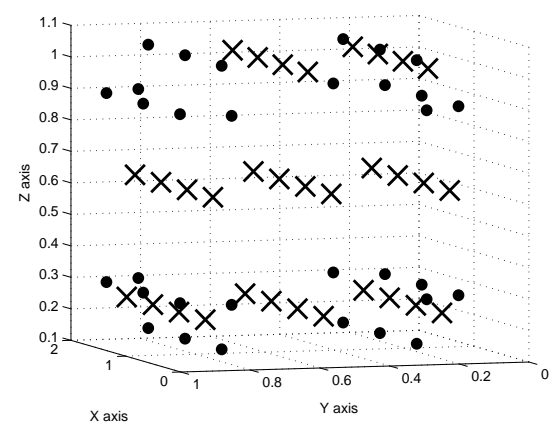

FiguRE 5 - Identification sources $(\times)$ and microphones $(\bullet)$

\begin{tabular}{|c|c|c|c|c|c|}
\hline Frequency $(\mathrm{Hz})$ & 50 & 130 & 250 & 330 & 450 \\
Index $E$ & $7.10^{-3}$ & 0.032 & 0.51 & 0.98 & 1.6 \\
\hline
\end{tabular}

TABLE 1 - Error index as a function of frequency

corners of the room where it goes down to $0.37 \mathrm{~m}$ ). The identification sources $S_{j}$ are located inside the volume bounded by the antenna of microphones. These sources represent a rectangular volume with boundaries situated at minimum distances of $0.33 \mathrm{~m}, 0.50 \mathrm{~m}$ and $0.23 \mathrm{~m}$ from the walls (along the $\mathrm{x}, \mathrm{y}, \mathrm{z}$-axis respectively). They are regularly spaced inside the volume because it is a convenient geometry for experimental conditions. However the spaces may differ of a few cms in order to avoid any specific data redundancy.

Figures 6 to 8 present the curves obtained for 3 frequencies 130, 250 and $330 \mathrm{~Hz}$. The abscissa corresponds to the 7 positions of the minimization microphones. In each figure, the upper part presents the comparison of the exact and estimated diffracted pressure levels. The continuous line represents the exact diffracted pressure level $p_{d i f}\left(\tilde{S}, M_{\ell}\right)$ and the crosses represent the estimated pressures $p_{\text {dest }}\left(\tilde{S}, M_{\ell}\right)$. The lower part presents a comparison of the exact and estimated phase of the diffracted pressure. The same scales are used for the 3 figures.

For the sound levels, at $130 \mathrm{~Hz}$, the estimation is quite close to the exact value. At $250 \mathrm{~Hz}$, the error goes from 0 to $2.5 \mathrm{~dB}$. At $330 \mathrm{~Hz}$, the error goes up to $10 \mathrm{~dB}$. In order to quantify the error, a global index has been defined as the mean value, over all points $M_{\ell}$, of the amplitude of the difference between the exact diffracted pressure and its estimated value. It increases from $7.10^{-3}$ at $50 \mathrm{~Hz}$ to 1.6 at $450 \mathrm{~Hz}$ as shown in Table 1 . As far as the phases are concerned, the comparison is correct at $130 \mathrm{~Hz}$. At $250 \mathrm{~Hz}$, the difference goes from 0 to 1.1 at the end points. At $330 \mathrm{~Hz}$, the difference is small at points 4 to 7 but goes up to $\pi$ for point 2 . For both levels and phases, the errors increase with frequency as expected. 

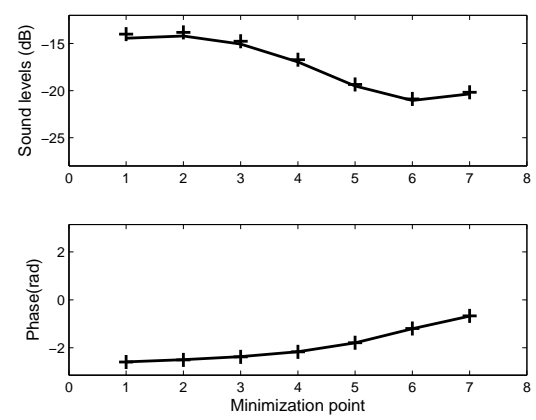

Figure 6 - Diffracted pressure levels and phases at $130 \mathrm{~Hz}$ - exact (line) and estimated $(+)$
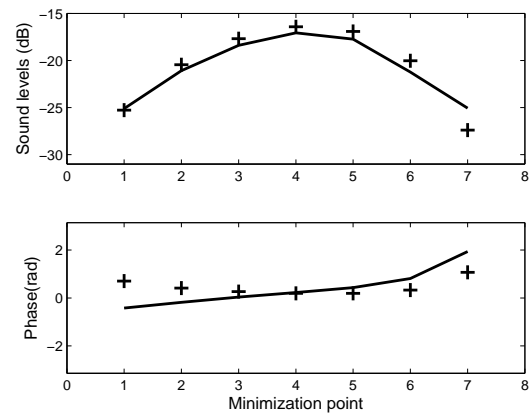

FiguRE 7 - Diffracted pressure levels and phases at $250 \mathrm{~Hz}$ - exact (line) and estimated (+)
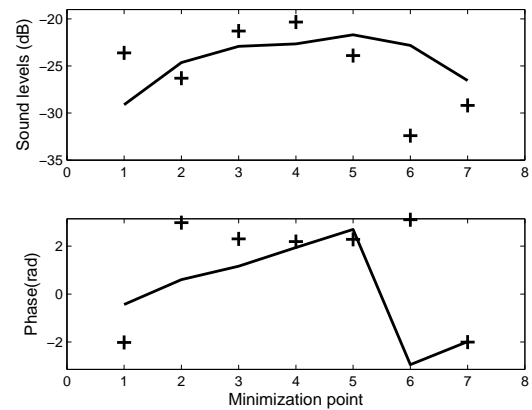

FiguRE 8 - Diffracted pressure levels and phases at $330 \mathrm{~Hz}$ - exact (line) and estimated $(+)$ 


\subsection{The control step}

The active control consists in estimating the control commands $u$ of the loudspeakers $V_{k}$ by minimizing the quantity :

$$
\begin{aligned}
J\left(M_{\ell}\right) & =\left\|p_{d i f}\left(\tilde{S}, M_{\ell}\right)+C_{t}\left(V_{k}, M_{\ell}\right) u\left(V_{k}\right)\right\|^{2} \\
& +\alpha\left\|u\left(V_{k}\right)\right\|^{2}
\end{aligned}
$$

where $C_{t}$ is the transfer matrix between the secondary sources $V_{k}$ and points $M_{\ell}$. The second term adds a condition on the norm of the solutions $u$ in order to get a well-posed problem even for very small $\alpha$. The minimization of $J\left(M_{\ell}\right)$ can be carried out with the exact value of the diffracted pressure $p_{\text {dif }}$ or with its estimated value $p_{\text {dest }}$. For the commands this leads to two minima called $u_{e x}$ and $u_{e s t}$ from which two approximations of $p_{d i f}$ are defined as follows :

$$
\tilde{p}_{d e x}=-C_{t} u_{e x} \quad \text { and } \quad \tilde{p}_{\text {dest }}=-C_{t} u_{e s t}
$$

The comparison between the two terms is a way to point out the influence of the identification step. The corresponding incident pressures are then estimated from the substraction between the exact total pressure and the estimated diffracted pressure. For example, $\tilde{p}_{\text {iest }}=p_{\text {tot }}-\tilde{p}_{\text {dest }}$. This is not a conventional way to present active control results. The idea here is to show the efficiency of the active control in relation with the respective levels of the incident and the diffracted pressures. As said before in the cases for which the diffracted pressure is quite small compared with the incident pressure, there is little interest for a high reduction of the diffracted pressure.

Figures 9 to 11 present the results obtained for the same 3 frequencies. The continuous curve represents the exact incident pressure $p_{i n c}\left(\tilde{S}, M_{\ell}\right)$ as a function of the 7 points $M_{\ell}$. For all frequencies the incident pressure $\tilde{p}_{i e x}$ is equal to the exact incident pressure which means that the control step itself works efficiently. This is not surprising since it consists in solving a problem with more unknowns than equations. The crosses + and $\times$ respectively represent the values of the estimated incident pressure $\tilde{p}_{\text {iest }}$ and the exact total pressure $p_{\text {tot }}$. The total pressure levels are shown in order to give the relative values of the diffracted pressure levels. Again the higher the diffracted pressure level is, the higher its estimate is crucial. In each figure, the two dotted lines are located $1.5 \mathrm{~dB}$ below and above the exact incident levels. This interval corresponds to the anechoicity criterion defined previously.

Figure 9 shows a good agreement between the sound levels of $p_{i n c}$ and $\tilde{p}_{\text {iest }}$. The difference between the total and the incident pressure levels points out that the diffracted pressure is a large part of the total pressure. This shows that the method (identification+control) provides a convenient tool at low frequency. At $250 \mathrm{~Hz}$ (figure 10) the agreement is still good except at point 7 (with a $5 \mathrm{~dB}$ deviation) and the diffracted pressure level is small compared with the total pressure. At $330 \mathrm{~Hz}$ (Figure 11) the method is not that efficient however the diffracted pressure level is much lower so that the accuracy on its estimation is not so critical. This shows that even if the accuracy of the results obtained in the identification step is not good the final result of the control step may still be satisfying. This points out that the identification criterion (used for the precalibration step) must be adapted to the final criterion.

In this example, most levels lie within the $3 \mathrm{~dB}$ interval around the exact incident pressure. This means that the method can be useful regarding the anechoicity standard rules. However this will not be sufficient in more specific studies such as the case of characterization measurements 


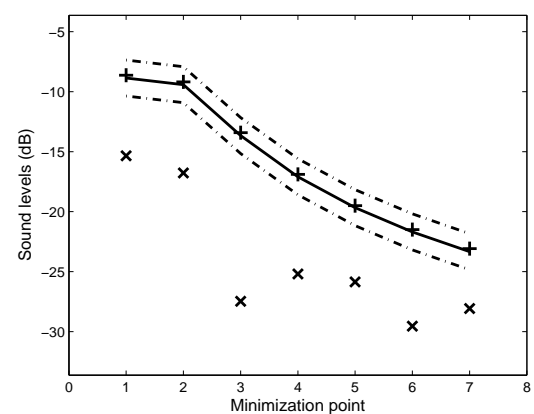

Figure 9 - Incident pressure levels at $130 \mathrm{~Hz}$ - exact (continuous line) and estimated (+), total pressure levels $(\times)$, the dotted lines represent a $3 \mathrm{~dB}$ interval around the exact incident pressure

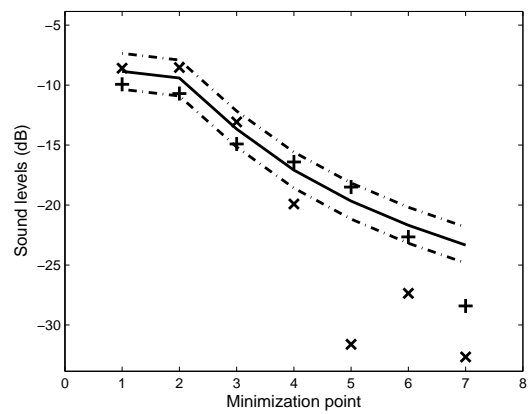

Figure 10 - Incident pressure levels at $250 \mathrm{~Hz}$ - exact (continuous line) and estimated (+), total pressure levels $(\times)$, the dotted lines represent a $3 \mathrm{~dB}$ interval around the exact incident pressure

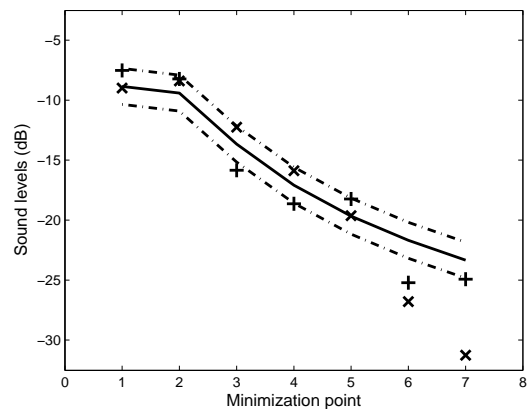

FIgURE 11 - Incident pressure levels at $330 \mathrm{~Hz}$ - exact (continuous line) and estimated (+), total pressure levels $(\times)$, the dotted lines represent a $3 \mathrm{~dB}$ interval around the exact incident pressure 
for the radiation of a source for which higher accuracy should be required. Further numerical studies must also be carried out to thoroughly determine the influence of the parameters and for more complex cases. For example, in realistic applications, active control may lead to errors if the number of minimization microphones is larger than the number of secondary sources.

\section{Conclusion}

In this paper, it has been shown that the total pressure measured on a closed surface is directly related to the diffracted pressure at any point inside this boundary. The proof of its existence relies on the mathematical study of a transmission problem. It has been shown that in the simple case of a half-space with an impedance condition, a formal explicit expression of the operator $\mathcal{H}$ can be obtained. This example is a convenient tool to further study the properties of non-smoothness of $\mathcal{H}$ which in turn provide information on the properties of the inverse problem and its regularization.

The relation between the total and the diffracted pressure provides a method to develop an active control system. The numerical tests presented were carried out for a scaled model on the frequency band $50-450 \mathrm{~Hz}$ in order to illustrate the method in the case of an anechoicity criterion.

Many questions still arise on numerical and experimental aspects. On the numerical side, the main points are the discretization of the formula and the regularization of the inverse problem. The parameters are the number and positions of the identification sources and microphones. Some results on compressive sensing [48] could provide some ideas on other choices of the sources location in order to obtain a better conditioning of the identification matrix. Possibly the directivity of the identification sources are also to be considered in order to improve the well-posedness of the problem. Let us underline that the essential criterion to be taken into account is the influence of the regularization of the identification problem on the active control performance rather than on the identification of the operator itself. On the experimental side, a first full-scale test is now carrying out in a semi-anechoic room with one highly reflecting vertical wall and five absorbing walls. The experiment will provide information on the best ways to associate identification and control and on the efficiency of the control.

Let us finally point out that this method can be useful for rooms other than anechoic and therefore for sound field synthesis.

\section{APPENDIX}

\section{A The half-space case - calculation of the operator $\mathcal{H}$}

The method follows [44]. The operator is obtained by inverting the Fourier transform through an integration in the complex plane. The integral to be calculated is given by :

$$
\mathcal{H}(\rho, z)=\frac{-\imath}{4} \int_{-\infty}^{\infty} \hat{\mathcal{H}}(\xi, z) H_{0}^{(1)}(\rho \xi) \xi d \xi
$$

It is well defined since the function to be integrated exponentially decreases at infinity and is properly defined at $\xi=0$ [43]. Following the principle of limit absorption (equivalent to Sommerfeld conditions [47]) it is assumed that the wave number $k$ has a small positive imaginary 
part and the final expression of $\mathcal{H}$ will be the limit of the result obtained by letting the imaginary part of $k$ tend to zero. The calculation below is therefore done with $k$ non real in which case the denominator of $\mathcal{H}$ has no zeros on the real axis.

In order to obtain layer potentials in the $(z=a)$ plane the first step is to introduce a term $e^{\imath K|z-a|}$ with :

$$
\hat{\mathcal{H}}=e^{\imath K|z-a|} \hat{F}\left(\xi^{2}\right)
$$

with

$$
\hat{F}\left(\xi^{2}\right)=\frac{e^{\imath K a}(K \zeta-k)}{2 K \zeta \cos K a-2 \imath k \sin K a}=e^{\imath K a} \frac{K \zeta-k}{2 Y\left(\xi^{2}\right)}
$$

Because of the square root $K$, the integration in the complex plane involves branch integrals. However as remarked in [44], these branch integrals disappear if the function to be integrated is even in $K$.

Therefore the function $\hat{F}$ is first written as :

$$
\hat{F}\left(\xi^{2}\right)=\frac{1}{2}+\hat{B}\left(\xi^{2}\right) \quad \text { with } \quad \hat{B}=\frac{-k \cos K a+\imath K \zeta \sin K a}{2 Y}
$$

where $\hat{B}$ is uneven in $K$. To obtain an even function one more step is introduced by using the following equalities :

$$
\begin{aligned}
\hat{B}\left(\xi^{2}\right) e^{\imath K|z-a|} & =\frac{-2}{\imath K} \hat{B}\left(\xi^{2}\right) \frac{-\imath K}{2} e^{\imath K|z-a|} \\
& =\frac{h_{b}\left(\xi^{2}\right)}{D\left(\xi^{2}\right)} \frac{-\imath K}{2} e^{\imath K|z-a|}
\end{aligned}
$$

where $D=K Y$ and $h_{b}=\imath(-k \cos K a+\imath K \zeta \sin K a)$. The function $h_{b} / D$ is an even function in $K$.

The next step is to compute the inverse Fourier transform of the two terms I and II defined by $\hat{\mathcal{H}}=I+I I$ with :

$$
I=\frac{1}{2} e^{\imath K|z-a|} \text { and } \quad I I=\frac{h_{b}\left(\xi^{2}\right)}{D\left(\xi^{2}\right)} \frac{-\imath K}{2} e^{\imath K|z-a|}
$$

For the first one let us remark that :

$$
I=\frac{\partial}{\partial z} \frac{e^{\imath K|z-a|}}{2 \imath K}
$$

so that the inverse Fourier transform is given by :

$$
\frac{\partial}{\partial z}\left(\frac{-e^{\imath k r}}{4 \pi r}\right)
$$

For the second term let us similarly remark that :

$$
\frac{-\imath K}{2} e^{\imath K|z-a|}=\frac{\partial^{2}}{\partial z \partial a}\left(\frac{e^{\imath K|z-a|}}{2 \imath K}\right)
$$

which is the Fourier transform of :

$$
\frac{\partial^{2}}{\partial z \partial a}\left(\frac{-e^{\imath k r}}{4 \pi r}\right)
$$


The Fourier transform of the function $h_{b} / D$ is obtained by the method of residues. The poles of this function, denoted $\lambda_{n}$, are the zeros of the denominator $D$. Let us assume for simplicity that these zeros are simple zeros, the integral can be written :

$$
-\frac{\imath}{2} \sum_{n} \frac{h_{b}\left(\lambda_{n}^{2}\right)}{D^{\prime}\left(\lambda_{n}^{2}\right)} \lambda_{n} H_{0}\left(\lambda_{n} \rho\left(P^{\prime}\right)\right)
$$

where $D^{\prime}\left(\lambda_{n}^{2}\right)=\partial D\left(\xi^{2}\right) / \partial\left(\xi^{2}\right)$ at $\xi=\lambda_{n}$.

The inverse Fourier transform of $I I$ is the convolution product of the terms in Equations (13) and (14). It is given by :

$$
\begin{aligned}
-\frac{\imath}{2} \frac{\partial}{\partial z} \int_{z^{\prime}=a} \sum_{n} \frac{h_{b}\left(\lambda_{n}^{2}\right)}{D^{\prime}\left(\lambda_{n}^{2}\right)} \lambda_{n} H_{0}\left(\lambda_{n} \rho\left(P^{\prime}\right)\right) \\
\\
\times \frac{\partial}{\partial z^{\prime}}\left(\frac{e^{\imath k r\left(Q, P^{\prime}\right)}}{4 \pi r\left(Q, P^{\prime}\right)}\right) d P^{\prime}
\end{aligned}
$$

with $Q=(x, y, z), P^{\prime}=\left(x^{\prime}, y^{\prime}, z^{\prime}\right)$.

The inverse Fourier transform of $\hat{\mathcal{H}}$ is the sum of Equation(12) and (15) which leads to the final expression (7).

\section{Références}

[1] S. Schneider, C. Kern : Acoustical behavior of the large anechoic chamber at the LMA in the low frequency range. Acta Acust united Ac 94 (2008) 141-147.

[2] M. Melon, C. Langrenne, D. Rousseau, Ph. Herzog : Comparison of four subwoofer measurement techniques. J. Audio. Eng. Soc., 55 (2007) 1077-1091.

[3] M. Melon, C. Langrenne, Ph. Herzog, A. Garcia : Evaluation of a method for the measurement of subwoofers in usual rooms. J. Acoust. Soc. Am., 127(2010) 256-263.

[4] C. Bellmann, W. Klippel, D. Knobloch : Holographic loudspeaker measurement based on near field scanning. DAGA Meeting 2015, Nuremberg.

[5] IEC 60268-5 International standard : Sound system equipment - Part 5 : Loudspeakers. Third edition 2003-05, International Electrotechnical Commission, Geneva, 2003.

[6] L.G. Beatty : Acoustic impedance in a rigid-walled cylindrical sound channel terminated at both ends with active transducers. J. Acoust. Soc.Am. 36 (1964) 1081-1089.

[7] D. Guicking, K. Karcher : Active impedance control for one-dimensional sound. J. Vibr. Acoust. 106 (1984) 393-396.

[8] F. Orduña-Bustamante, P.A. Nelson, An adaptive controller for the active absorption of sound. J. Acoust. Soc. Am. 91 (1992) 2740-2747.

[9] R.L. Clark D.G. Cole, Active damping of enclosed sound fields through direct rate feedback control. J. Acoust. Soc. Am. 97 (1995) 1710-1716.

[10] D. Thenail, O. Lacour, M.A. Galland, M. Furstoss : The active control of wall impedance. Acta Acust united Ac 83 (1997) 1039-1044.

[11] H. Zhu, R. Rajamani, K.A. Stelson : Active control of acoustic reflection, absorption and transmission using thin panel speakers. J. Acoust. Soc. Am. 113 (2003) 852-870. 
[12] H. Lissek, X. Meynial : A preliminary study of an isodynamic transducer for use in active acoustic materials. Appl. Ac. 64 (2003) 917-930.

[13] J. Yuan : Causal impedance matching for broadband hybrid noise absorption. J. Acoust. Soc. Am. 113 (2003) 3226-3232.

[14] D. Guicking, E. Lorentz : An active sound absorber with porous plate. J. Vibr. Acoust. 106 (1984) 389-392.

[15] S. Beyene, R.A. Burdisso : A new hybrid passive/active noise absorption system. J. Acoust. Soc. Am. 101 (1997) 1512-1515.

[16] D. Thenail, M.A. Galland, M. Sunyach, M. Sunhack : Active enhancement of the absorbent properties of a porous material. Smart Mater. Struct. 3 (1994) 18-25.

[17] J.P. Smith, B.D. Johnson, R.A. Burdisso : A broadband passive-active sound absorption system. J. Acoust. Soc. Am. 106 (1999) 2646-2652.

[18] P. Cobo, J. Pfretzschner, M. Cuesta, D.K. Anthony : Hybrid passive-active absorption using microperforated panels. J. Acoust. Soc. Am. 116 (2004) 2118-2125.

[19] P. Leroy, A. Berry, Ph. Herzog, N. Atalla : Experimental study of a smart foam sound absorber. J. Acoust. Soc. Am., 129 (2011) 154-164.

[20] M. Melon, Ph. Herzog, A. Sitel, M.A. Galland : One dimensional study of a module for active/passive control of both absorption and transmission. Applied Acoustics 73 (2012) $234-242$.

[21] T.W. Leishman, J. Tichy : On the significance of reflection coefficients produced by active surfaces bounding one-dimensional sound fields. J. Acoust. Soc. Am. 113 (2003) 1475-1482.

[22] D. Guicking, K. Karcher : Coherent active methods for applications in room acoustics. J. Acoust. Soc. Am. 78 (1985) 1426-1434.

[23] Ph. Herzog, A. Soto-Nicolas, F. Guéry. Passive and active control of the low-frequency modes in a small room. 98th Audio Eng. Soc. Convention, number 3591 :1-12, Paris, 1995.

[24] L. Nicolas, M. Furtoss, M.A. Galland : Analogy electromagnetism-acoustics : Validation abd application to local impedance active control for sound absorption. Eur. Phys. J. AP (1998) 95-100.

[25] O. Lacour, M.A. Galland, D. Thenail : Preliminary Experiments on Noise Reduction in Cavities Using Active Impedance Changes. J. Sound Vib. 230 (2000) 69-99.

[26] E. Friot, A. Gintz, Ph. Herzog, S. Schneider : Improving absorption of sound using active control. Motion and vibration control : Selected Papers from MOVIC 2008, 83-91, Springer, 2009 .

[27] P.A. Nelson, S.J. Elliott : Active Control of Sound. Academic Press, London, 1993 (ch. 9 : Active suppression of scattered acoustic radiation).

[28] Y. I. Bobrovnitskii : A new-impedance based approach to analysis and control of sound scattering. J. Sound Vib. 297 (2006) 743-760.

[29] D.A.B. Miller : On perfect cloaking. Optics Express 14 (2006) 12457-12466.

[30] E. Friot, R. Guillermin, M. Winninger : Active control of scattered acoustic radiation : A real-time implementation for a 3D object. Acta Acust united Ac 92 (2006) 278-288.

[31] N. Han, S. Feng, X. Qiu : Active control of one-dimension impulsive reflection based on a prediction method. J. Acoust. Soc. Am. 127 (2010) 1193. 
[32] N. Han, X. Qiu : Active control on the scattered radiation by a rigid surface. J. Acoust. Soc. Am., 131 (2012) 3471.

[33] E. Friot : Control of low-frequency wall reflections in an anechoic room. Active 2006, Adelaide (Australia), 2006.

[34] E. Friot, A. Gintz : Estimation and global control of noise reflections. Active 2009, Ottawa (Canada), 2009.

[35] Ph. Herzog, E. Friot, D. Habault, C. Pinhede, A. Gintz, P. Leroy, M. Pachebat : Towards an active anechoic room. Proc. Forum Acusticum, Krakow (Poland), 2014.

[36] C. Pinhede, E. Friot, D. Habault, Ph. Herzog : Contrôle actif/passif de salle anéchoïque [Passive/active control for anechoic room]. Proc. Congrès Français d'Acoustique, Le Mans (France), 2016.

[37] D. Colton, R. Kress : Inverse acoustic and electromagnetic scattering theory. Applied Math Sciences 93, Wiley, 1983.

[38] P.J.T. Filippi : Problème de transmission pour l'équation de Helmholtz scalaire et problèmes aux limites équivalents : application à la transmission gaz parfait - milieu poreux. Jal de Mécanique, 18 (1979) 565-591.

[39] R.E. Kleinmann, P.A. Martin : On single integral equation for the problem of transmission in Acoustics. SIAM Jal Appl.Math. 48 (1988) 307-325.

[40] T.S. Angell, R.E. Kleinmann : $L^{2}$-boundary integral equations for the Robin problem. Math.Meth. in the Appl.Sci., 6 (1984) 345-352.

[41] Ph. Morse, H. Feshbach : Methods of Theoretical Physics. McGraw-Hill, 1953.

[42] X. Antoine, M. Darbas : Integral Equations and Iterative Schemes for Acoustic Scattering Problems. hal-00591456, to appear.

[43] M.Abramowitz, I.A. Stegun : Handbook of mathematical functions. Dover Pub. NewYork, 1972.

[44] D.Habault, P.J.T. Filippi : On the resolvent of the Pekeris operator with a Neumann condition. J. Sound Vib. 56 (1978) 87-95.

[45] S. Elliott : Signal processing for active control. Academic Press, London 2001.

[46] C. Hansen : Discrete Inverse Problems - Insight and algorithms. SIAM Philadelphia, 2010.

[47] B.R. Vainberg : Principles of radiation, limit absorption and limit amplitude in the general theory of partial differential equations. Russian Mathematical Surveys, 21 (1966) 115-193.

[48] G. Chardon, L. Daudet, A. Peillot, F. Ollivier, N. Bertin, R. Gribonval : Near-field acoustic holography using sparse regularization and compressive sampling principles. J.Acoust Soc Am., 132 (2012) 1521-1534. 